\title{
Tempo de jejum dos suínos no manejo pré-abate sobre a perda de peso corporal, o peso do conteúdo estomacal e a incidência de úlcera esofágica-gástrica
}

\author{
Relation of fasting time during pre-slaughter management to weight loss, weight of stomach \\ contents and incidence of gastric ulcer in pigs
}

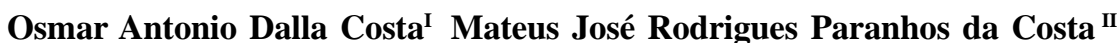 \\ Jorge Vitor Ludke ${ }^{\text {III }}$ Arlei Coldebella ${ }^{\text {III }}$ Jalusa Deon Kich ${ }^{\text {III }}$ \\ José Vicente Peloso $^{\text {IV }}$ Luigi Faucitanov ${ }^{\text {V }}$ Darlan Dalla Roza $^{\text {VI }}$
}

\section{RESUMO}

\begin{abstract}
O objetivo deste trabalho foi avaliar os efeitos do tempo de jejum (9, 12, 15 e 18 horas) dos animais na granja (TJG) e da posição na carroceria do caminhão (PBO) durante o transporte da granja ao frigorífico sobre: a perda de peso corporal (PPC), o peso do estômago cheio (PEC) e vazio (PEV), o peso do conteúdo estomacal (PCE) e o escore de lesão na mucosa esofágica-gástrica (ELG). Foram utilizadas 192 fêmeas, com peso vivo médio de $134,51 \pm 11,80 \mathrm{~kg}$. No modelo, foram considerados os efeitos de bloco (estação do ano, BL), TJG, PBO e da interação entre BL x TJG. Verificouse efeito significativo do TJG apenas sobre o peso do conteúdo estomacal. Não se observou efeito significativo da PBO sobre qualquer das variáveis avaliadas. Dos suínos avaliados, 90,3\% apresentaram PEC menor que 500 gramas e 8,56\% com PEC entre 500 e 800 gramas. A prevalência de suíno com ELG foi baixa (14,97\%), sendo que, dos animais com ELG, 13,90\% apresentaram lesão de grau 1 e 1,09\% eram grau dois. Concluise que animais submetidos a jejum na granja de 15 horas apresentam menor peso do conteúdo estomacal ao abate.
\end{abstract}

Palavras-chave: tempo de jejum, manejo pré-abate, peso de estômago, rendimento ao abate, suínos.

\section{ABSTRACT}

The objective of this study was to evaluate the effect of fasting time period (9,12, 15 and 18 hours) at the farm (TJG) and the pig's position in the lorry's livestock compartment (PBO) during the transport from the farm to the processing plant on: body weight loss (PPC), weight of stomach (full= $P E C$ and empty $=P E V)$, weight of stomach contents (PCE), and incidence of gastric ulcer (ELG). A total of 192 females with average $34.51 \pm 11.80 \mathrm{~kg}$ of body weigh were used. The statistical model considered the effects of: block (season, $B L$ ), TJG, PBO and the interaction between BL and TJG. There was a significant effect of TJG only on stomach content. There was no effect of $P B O$ on any of the evaluated variables. A PEC inferior to $500 \mathrm{~g}$ was observed in $90.3 \%$ of the animals, while $8.56 \%$ of the pigs presented PEC between 500 and $800 \mathrm{~g}$. Incidence of ELG was low (14.97\%), and among the ELG animals, $13.90 \%$ showed grade 1 lesion, and $1.09 \%$ grade two lesion. As a conclusion, pigs submitted to a fasting period of 15 hours have less stomach contents at the processing.

Key words: fasting time, pre-slaughter management, carcass yield, stomach weight, swine.

\section{INTRODUÇÃO}

Ojejum pré-abate é realizado no período final da fase de terminação de suínos e dura até o abate. Nesse período, os animais permanecem sem acesso a alimentos sólidos (ração), mas devem ter livre acesso à água. Esse manejo é de grande importância para o produtor de suínos e para os abatedouros, pois contribui para: economia de ração, redução da taxa de mortalidade durante o transporte, aumento da velocidade e facilidade no processo de evisceração, redução no volume de dejetos e padronização no rendimento das carcaças e da qualidade da carne

IEmbrapa Suínos e Aves, BR 153, Km 110, CP 21, 89700-000, Concórdia, SC, Brasil. Programa de Pós-graduação em Zootecnia (Produção Animal), Faculdade de Ciências Agrárias e Veterinárias (FCAV), Universidade Estadual Paulista (UNESP). E-mail: osmar@cnpsa.embrapa.br. Autor para correspondência.

IIDepartamento de Zootecnia, Grupo de Estudos e Pesquisas em Etiologia e Ecologia Animal (ETCO), FCAV/UNESP, São Paulo, SP, Brasil.

IIIEmbrapa Suínos e Aves, Concórdia, SC, Brasil.

Iv Sadia S. A., Concórdia, Sc, Brasil.

${ }^{v}$ Agriculture and Agri-Food Canada, Dairy and Swine Research and Development Centre, Lennoxville, Quebec, Canada.

VITRIEL-HT Industria de Equipamentos Rodoviários Ltda, Erechim, Rs, Brasil. 
(TARRANT, 1991; BEATTIE et al., 2002; DALLA COSTA et al., 2006).

Na literatura consultada, verificou-se grande variação quanto às recomendações do tempo de jejum dos suínos na granja. Por exemplo, MURRAY (2000) recomendou entre 10 e 24 horas, CHEVILLON (1994) de 12 a 18 horas e EIKELENBOON et al. (1991) de 16 a 24 horas. MAGRAS et al. (2000) sugeriram tempo de jejum mais longo (de 22 a 28 horas), objetivando obter maior porcentagem de suínos com menor peso estomacal $(<1,4 \mathrm{~kg})$. Essa grande variação na recomendação do tempo de jejum dos suínos na granja está relacionada ao sistema de logística das granjas e dos frigoríficos.

Há evidências de que o jejum dos suínos na granja resulta em perdas qualitativas e quantitativas na carne. Suínos submetidos a 24 horas de jejum podem perder até $5 \%$ de seu peso corporal, a uma taxa de $0,20 \%$ por hora, sendo que essas perdas podem ser maiores quanto mais pesados forem os animais. Por exemplo, indivíduos com 71,0kg de peso submetidos a jejum de 48 horas podem perder até $7,1 \%$ do peso (ou $0,11 \mathrm{~kg} /$ hora) (MURRAY \& JONES, 1992; BEATTIE et al., 1999). Suínos abatidos com $100 \mathrm{~kg}$ de peso podem perder $5 \mathrm{~kg}$ após jejum de 24 horas, sendo que a perda de peso na carcaça pode variar de 20 a $31 \%$ do peso vivo quando submetidos a jejum de 24 e 48 horas, respectivamente (JONES et al., 1985; 1988; EIKELENBOOM et al., 1991). JONES et al. (1985) relataram que $80 \%$ da perda de peso ocorre nas primeiras 24 horas do jejum.

O objetivo deste trabalho foi avaliar o efeito de certas práticas do manejo pré-abate (tempo de jejum dos suínos na granja e de sua posição na carroceria do caminhão durante o transporte da granja até o frigorífico) sobre a perda de peso corporal (PPC), do peso do estômago cheio (PEC), vazio (PEV) e do peso do conteúdo do estômago (PCE) e na incidência de úlcera esofágica-gástrica (LEG).

\section{MATERIAL E MÉTODOS}

Foram utilizadas 192 fêmeas suínas oriundas de cruzamentos industriais, com peso médio de $134,51 \pm 9,75 \mathrm{~kg}$ no inverno e de $131,75 \pm 11,80 \mathrm{~kg}$ no verão. Nas duas granjas avaliadas, foram escolhidas aleatoriamente quatro baias por tempo de jejum dos suínos na granja com seis animais por baia, totalizando 16 baias para a realização do experimento e 96 suínos por granja. No manejo pré-abate, os suínos receberam tempos de jejum na granja antes do carregamento de 9 , 12, 15 ou 18 horas. Os suínos foram pesados uma hora após a última refeição e imediatamente antes do abate para a obtenção da perda de peso corporal (PPC). Os animais foram transportados em um modelo de carroceria metálica dupla, com capacidade de transporte de 96 suínos.

A insensibilização via eletronarcose foi aplicada automaticamente, transferindo alta voltagem (700V) e amperagem acima de 1,25Amps (VALHALLA, STORK RMS b.v., LICHTENVOORDE, Holanda). Após a insensibilização, os animais eram imediatamente sangrados na posição horizontal e suspensos ao fim da mesa de sangria contínua na linha de abate. Durante o processo de evisceração dos animais em avaliação, coletou-se o estômago, o qual foi pesado cheio (PEC) e vazio (PEV) - após a retirada do conteúdo estomacal e limpeza com água corrente, pesando-se também o conteúdo estomacal (PCE), classificado em quatro categorias, conforme CHEVILLON (1994). Após a limpeza dos estômagos, os mesmos foram examinados macroscopicamente para avaliação da presença de úlcera esofágica-gástrica (LEG), seguindo classificação segundo MORÉS et al. (2000).

As carcaças dos suínos permaneceram em câmara fria submetidas a temperaturas variando entre $1 \mathrm{e} 4^{\circ} \mathrm{C}$ por 24 horas, e as medidas de $\mathrm{pH}$ foram realizadas na meia-carcaça esquerda nos músculos Longissimus dorsi (LD) e Semimembranosus (SM), 24 horas após o abate $\left(\mathrm{pH}_{\mathrm{U}}\right)$. $\mathrm{Na}$ avaliação do $\mathrm{pH}$, foi utilizado o pHmetro portátil da marca Mettler Toledo (MP $120 \mathrm{pH}$ Meter, Suíça) com eletrodo DXK-S7/25 protegido para inserção no músculo.

$\mathrm{Na}$ análise das variáveis, utilizou-se o método de quadrados mínimos, aplicando-se os procedimentos GLM do programa Statistical Analysis System (SAS, 2001), com o seguinte modelo matemático:

$$
\mathrm{Y}_{\mathrm{ijklmn}}=\mu+\mathrm{BL}_{\mathrm{i}}+\mathrm{TJG}_{\mathrm{j}}+(\mathrm{BL} \times \mathrm{TJG})_{\mathrm{ij}}+\mathrm{PBO}_{\mathrm{k}}+
$$
$\mathrm{PPI}_{1}+\mathrm{PLA}_{\mathrm{m}}+\mathrm{COV}+\mathrm{e}_{\mathrm{ijk} k \mathrm{mn}}$, sendo $\mathrm{Y}_{\mathrm{ijk} k m n}=\mathrm{PPC}, \% \mathrm{PPC}$, PEC, PEV, PCE; $\mu=$ média geral; $\mathrm{BL}_{\mathrm{i}}=$ bloco (estação do ano, $\mathrm{i}=1$ inverno e 2 verão); $\mathrm{TJG}_{\mathrm{j}}=$ tempo de jejum dos suínos na granja antes do carregamento, $\mathrm{j}=1,2,3$, 4 (tempo de jejum de 9, 12, 15, 18 horas, respectivamente) $\mathrm{PBO}_{\mathrm{k}}=$ posição do animal na carroceria, $\mathrm{k}=1$ (frente), 2 (meio) e 3 (atrás); $\mathrm{PPI}_{1}=$ piso da carroceria, $1=1$ (inferior) e 2 (superior); $\mathrm{PLA}_{\mathrm{m}}=$ lado da carroceria, $\mathrm{m}=1$ (direto) e 2 (esquerdo); $(\mathrm{BL} \times \mathrm{TJG})=$ interação entre bloco e tempo de jejum dos suínos tha granja antes do carregamento; $\mathrm{COV}=$ covariável de interesse para estudo da variável dependente (perda de peso durante o manejo pré-abate ajustada pelo peso dos suínos na granja antes do carregamento); $\mathrm{e}_{\mathrm{ijk} k \mathrm{mn}}=$ erro aleatório. de Qui-Quadrado.

A incidência de LEG foi analisada pelo Teste

\section{RESULTADOS E DISCUSSÃO}

Não houve efeito significativo do tempo de jejum sobre $\mathrm{PPC}(\mathrm{P}=0,1515)$ e $\% \mathrm{PPC}(\mathrm{P}=0,1239)$, como caracterizado na figura 1 . Uma das razões por não ter 

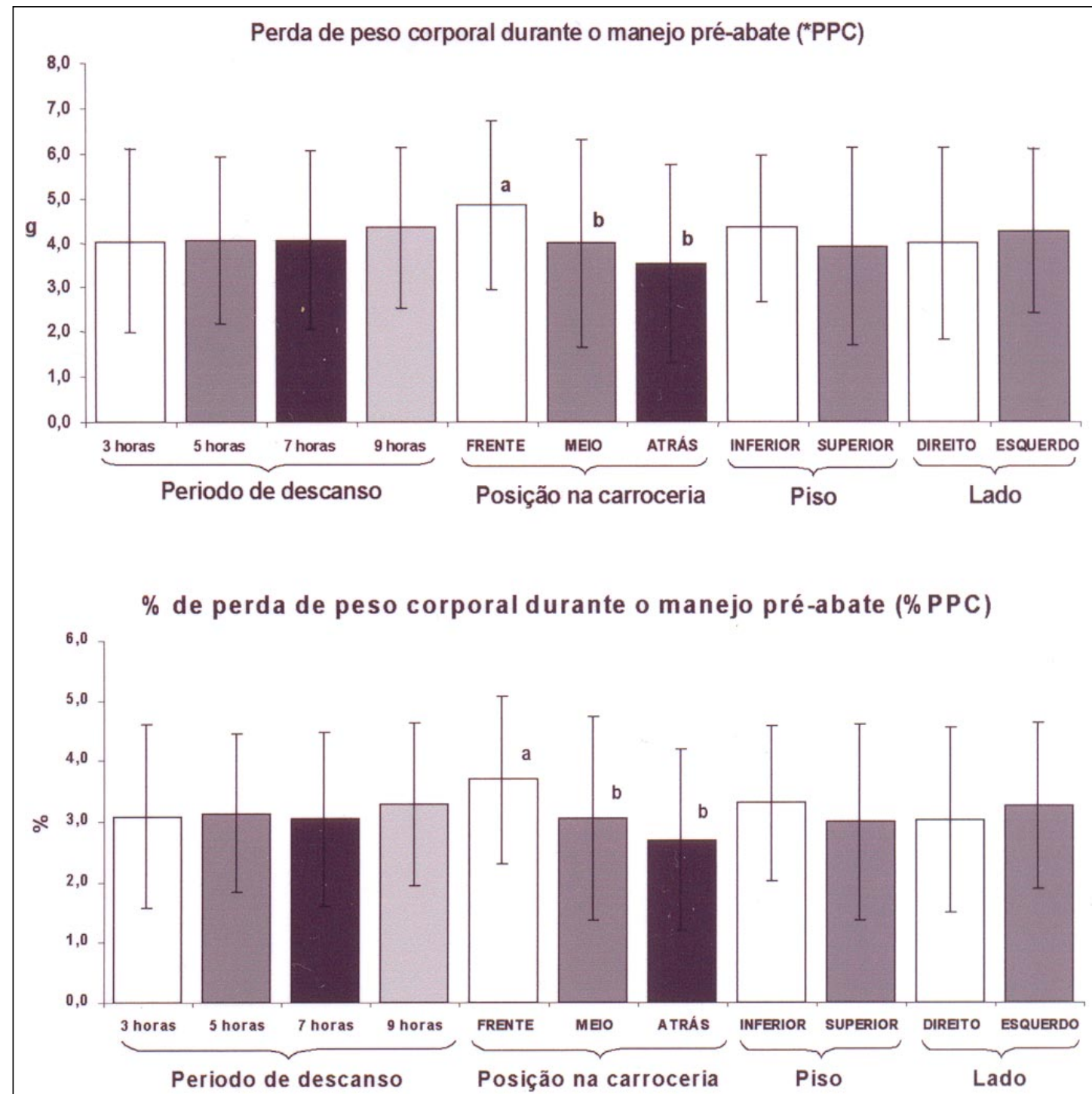

Figura 1 - Médias ajustadas e desvios-padrão da perda de corporal peso (PPC)* e da porcentagem (\%PPC) em função do tempo de jejum adotado e da posição dos animais na carroceria do caminhão. * Variável ajustada pelo peso do suíno na granja antes do carregamento $(\mathrm{P}=0,8001)$.

sido observado efeito do tempo do jejum na perda de peso durante o manejo pré-abate pode ter sido a duração do jejum, que foi inferior ao tempo avaliado em outros estudos.

Resultados prévios (WARRISS, 1982; WARRISS \& BROWN, 1983; JONES et al., 1988, BIDNER, 1999) demonstraram que o jejum pré-abate (0 a $60 \mathrm{~h}$ ) influenciou significativamente no rendimento de carcaça dos suínos. Para amenizar a perda de peso no manejo pré-abate, WARRISS (1982) indicou jejum de 18 horas (o tempo máximo avaliado no presente estudo) e sugeriu que o tempo de jejum na granja deve variar em função da duração do transporte, do período de descanso no abatedouro e das condições do manejo pré-abate.

A localização do box, do piso e do lado dentro da carroceria não influenciaram significativamente a $\mathrm{PPC}(\mathrm{P}=0,1884 ; \mathrm{P}=0,8222$ e $\mathrm{P}=0,9969)$ e a $\% \mathrm{PPC}(\mathrm{P}=0,1239, \mathrm{P}=0,1970$ e $\mathrm{P}=0,7787)$. Os resultados obtidos no presente estudo diferem dos obtidos por DALLA COSTA et al. (2006), que observaram efeito da posição do box da carroceria sobre a PPC e \%PPC; todavia, esses pesquisadores não encontraram efeito do período de descanso no frigorífico, piso e lado da carroceria sobre essas variáveis. 
O tempo de jejum dos suínos na granja não influenciou no $\mathrm{PEC}(\mathrm{P}=0,0802)$ nem no $\mathrm{PEV}(\mathrm{P}=0,9258)$. Contudo, teve efeito significativo no $\mathrm{PCE}(\mathrm{P}=0,0328)$, havendo diminuição do conteúdo estomacal dos suínos em função do aumento do tempo de jejum na granja.
Os animais que receberam jejum de 15 e 18 horas apresentaram menores valores de PCE, não havendo diferença significativa entre estes dois tratamentos (Figura 2). Esses resultados validam os obtidos por DALLA COSTA et al. (2006), que verificaram uma
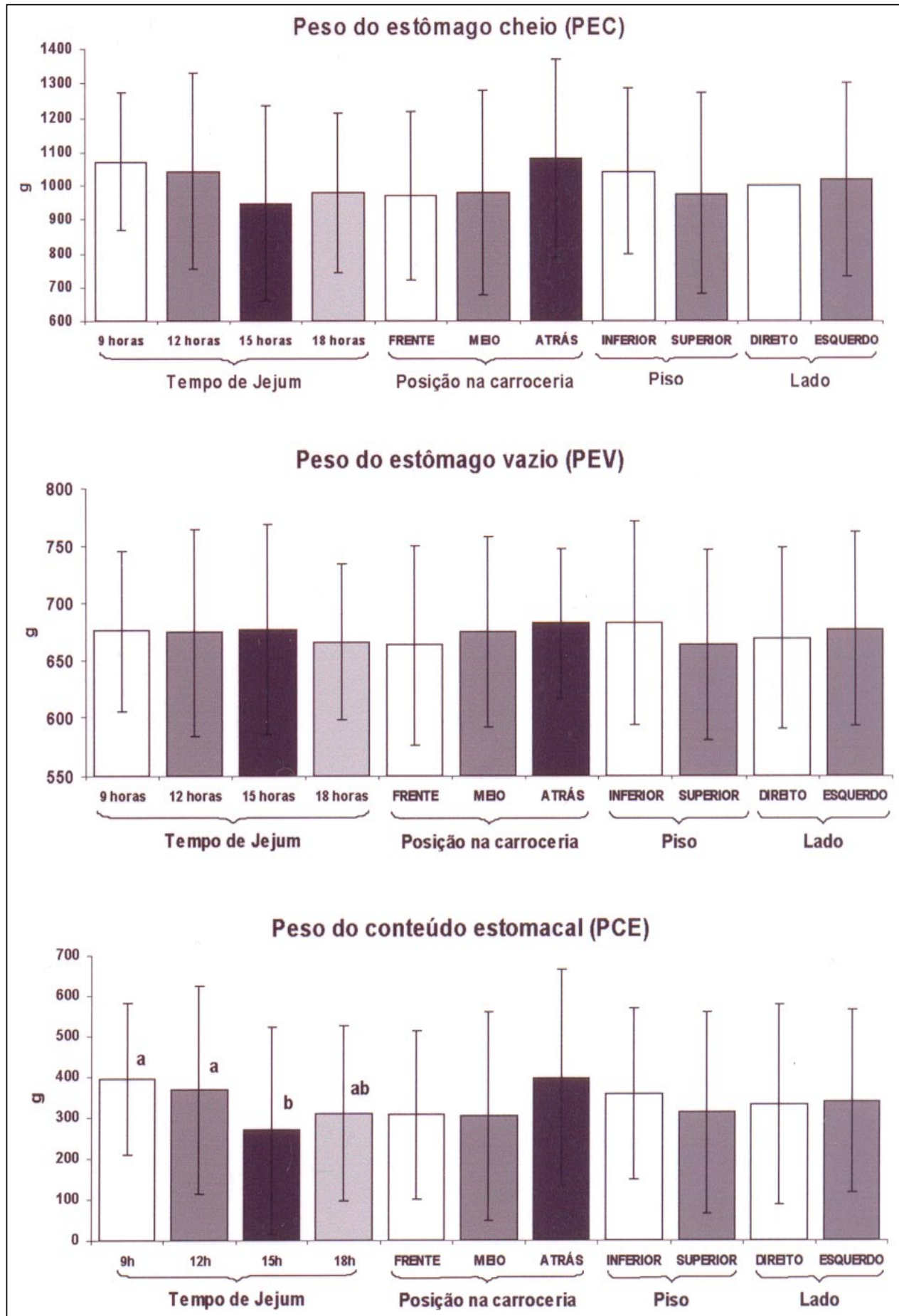

Figura 2 - Médias ajustadas e desvios-padrão do peso de estômago cheio, (PEC), vazio (PEV) e conteúdo estomacal (PCE) por tempo de jejum dos suínos na granja, e da posição do box dentro da carroceria do caminhão. Médias seguidas de letras minúsculas distintas dentro de cada fator diferem significativamente pelo teste $\mathrm{T}(\mathrm{P}<0,05)$. 
redução no PCE dos suínos com o incremento do tempo de jejum na granja.

Estudos realizados por LAWRENCE et al. (1998) encontraram efeito do jejum pré-abate em PEC e PCE, e suínos que não foram submetidos a jejum nesta fase da produção apresentaram valores significativamente maiores de PEC e PCE em relação aos que foram submetidos a jejum de 24 horas. Esses pesquisadores não encontraram efeito do jejum dos suínos durante o manejo pré-abate sobre o PEV.

Com relação ao PCE, pode-se concluir que os suínos foram submetidos a períodos de jejum adequados, pois, no presente estudo, não foi encontrado nenhum estômago com PCE maior que $1.100 \mathrm{~g}$ (indicativo de suínos com estômago cheio). Dos animais avaliados com presença de conteúdo estomacal, 169 suínos (90,37\%) apresentaram PCE menor que $500 \mathrm{~g}$ (indicativo de suínos com estômagos vazios). Observou-se baixa incidência de suínos $(8,56 \%)$ com PCE entre 500-800g (tendência a estômago vazio), e somente dois animais $(1,07 \%)$ apresentaram PCE entre $>800-1100 \mathrm{~g}$ (com tendência a estômago cheio). Estudo realizado por LYNCH et al. (2004) em dois frigoríficos mostrou resultado semelhante, com a grande maioria dos suínos avaliados apresentando PCE menor que $400 \mathrm{~g}$ (61 e $54 \%$ respectivamente) e somente 6 e $10 \%$ dos suínos observados apresentando PCE maior que $1000 \mathrm{~g}$.

Os resultados obtidos no presente estudo também validam os resultados de MURRAY et al. (2001), que encontraram alta porcentagem ( 82 e $72 \%$ ) de suínos com PCE menor que $500 \mathrm{~g}$ em animais submetidos a jejum de 15 horas no frigorífico ou na granja, respectivamente. Os valores médios do PCE foram menores que os encontrados por CHEVILLON (1994), GUISE et al. (1995) e LYNCH et al. (2004). Essas diferenças podem ser atribuídas aos diferentes procedimentos aos quais os suínos foram submetidos durante o manejo pré-abate, tais como o tempo de jejum, a duração do transporte, o descanso e o sistema de alimentação).

A localização do box, piso e lado da carroceria não influenciaram significativamente a $\mathrm{PEC}$ $(\mathrm{P}=0,0598 ; \mathrm{P}=0,0781$ e $\mathrm{P}=0,6557)$, o $\mathrm{PEV}(\mathrm{P}=0,5213$; $\mathrm{P}=0,1190$ e $\mathrm{P}=0,4889)$ e o $\mathrm{PCE}(\mathrm{P}=0,0557 ; \mathrm{P}=0,1454$ e $\mathrm{P}=0,7989)$. Esses resultados validam os obtidos por DALLA COSTA et al. (2006), que também não encontraram efeito no manejo pré-abate sobre essas variáveis.

A úlcera esofágica-gástrica (LEG) em suínos tem etiologia complexa e múltipla, e é por isso considerada uma doença multifatorial (MORÉS, 1998). No presente estudo, o tempo de jejum dos suínos na granja não influenciou significativamente $(\mathrm{P}=0,2750)$ a incidência de LEG (Figura 3), que pode ser considerada como baixa (14,97\%), sendo que, $13,90 \%$ (26 suínos) desses animais apresentaram escore de úlcera um e somente 1,09\% (2 suínos) apresentaram úlcera com escore dois. A incidência de animais sem LEG e com paraqueratose foi alta (48,13 e 36,90\%, respectivamente). Esses resultados estão de acordo com os obtidos por EISEMANN et al. (2002), que também não encontraram efeito significativo do jejum ( 0,12 e 24 horas) pré-abate sobre a incidência de LEG dos suínos. Contudo, diferem dos encontrados por LAWRENCE et al. (1998), segundo os quais houve um efeito do jejum pré-abate sobre o escore de LEG, e suínos que permaneceram em jejum por 24 horas apresentaram escores de LEG significativamente

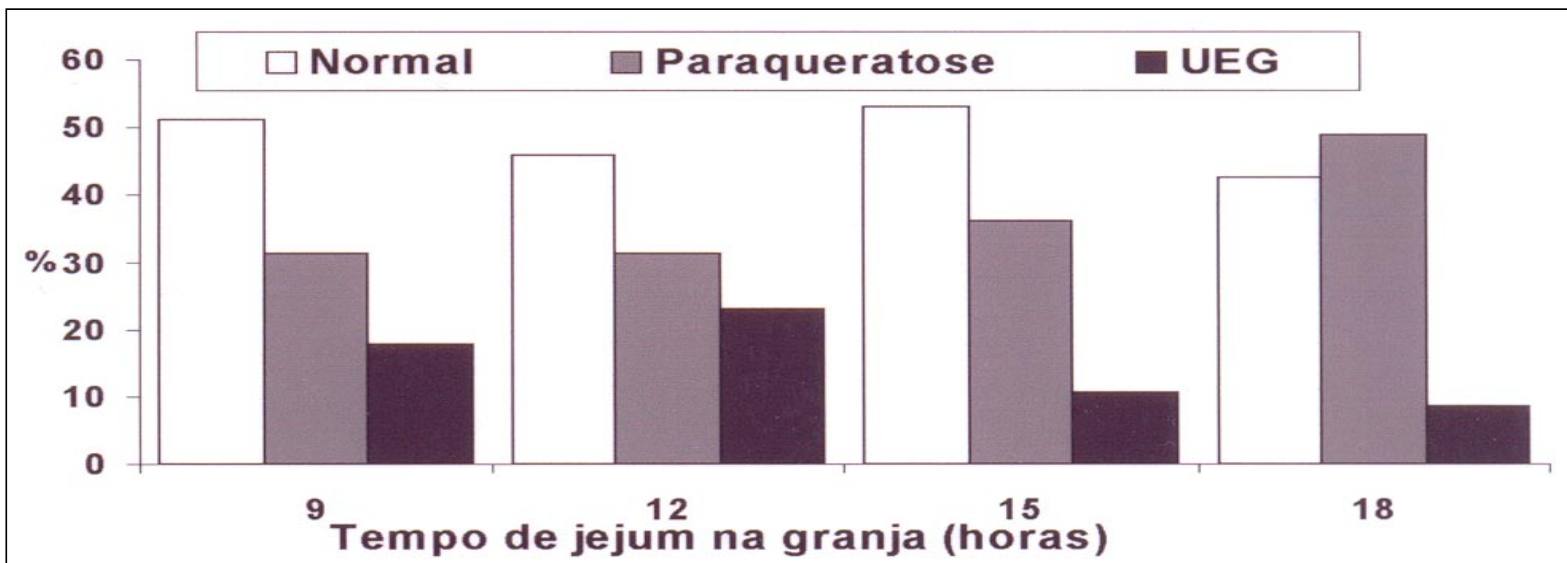

Figura 3 - Média da porcentagem de suínos com estômago normal (sem lesão), com paraqueratose (PQ) e com úlcera esofágicagástrica (LEG) em função do tempo de jejum dos suínos na granja. 
maiores em relação aos que não foram submetidos a jejum (1,65 e 0 respectivamente). BIDNER (1999) também encontrou efeito significativo do tempo de jejum (12, 36 e 60 horas) dos suínos no escore de LEG $(0,0,96$ e $1,44)$, sendo que $79 \%$ dos suínos que foram submetidos a jejum de 36 horas apresentaram problemas de paraqueratose. Suínos com jejum de 60 horas apresentaram menor incidência de paraqueratose (57\%) e maior porcentagem de estômagos com úlcera (43\%). DALLA COSTA et al. (2006), estudando o período de descanso no frigorífico, também não encontraram efeito do manejo pré-abate sobre a LEG.

Estudos realizados por MORROW et al. (2004) também encontraram efeito significativo do jejum pré-abate $(0,12$ e 24 horas) sobre a incidência de LEG $(3,8,3,6$ e 4,1 , respectivamente).

Os resultados encontrados no presente estudo mostram que o jejum de 14 a 23 horas durante o manejo pré-abate (jejum na granja de 9 a 18 horas, descanso no frigorífico de três horas e duas horas para o embarque, transporte e desembarque) não é um período suficiente para o surgimento de uma LEG nos suínos, porém pode contribuir para a incidência de aumento de estômagos com paraqueratose.

$\mathrm{Na}$ figura 4, são apresentadas as classificações das carcaças em relação ao $\mathrm{pH}_{U}$ dos músculos Longissimus dorsi (LD) e semimembranosus ( $\mathrm{SM})$ : baixo $(=5,55)$, bom $(5,55<\mathrm{pHU}=5,90)$ e elevado $(>5,90)$, em relação a estômago normal e com úlcera esofágica-gástrica (LEG).
A presença de LEG não influenciou significativamente $(\mathrm{P}=0,1698)$ a classificação das carcaças em função do pHU do músculo SM. Todavia, observou-se efeito significativo $(\mathrm{P}=0,0336)$ da presença de LEG na classificação das carcaças em relação ao pHU do músculo do LD. Dos suínos com LEG, 57,14\% apresentaram carcaças com baixo $\mathrm{pHU}(=5,55)$ e $42,86 \%$ com pHU bom $(5,55<\mathrm{pHU}=5,90)$. Dos animais com estômagos normais, $31,85 \%$ das carcaças apresentavam pHU baixo $(=5,55)$, sendo que a grande maioria desses suínos $(66,88 \%)$ apresentou carcaças com pHU bom $(5,55<\mathrm{pHU}=5,90)$ e somente $1,27 \%$ apresentaram carcaças com pHU elevado $(>5,90)$.

\section{CONCLUSÕES}

Os tempos de jejum dos suínos na granja avaliados no presente estudo não promoveram maior perda de peso, nem apresentaram efeito sobre a incidência de úlcera esofágica-gástrica (LEG). Todavia, o incremento do jejum na granja para os animais contribuiu na redução do peso do conteúdo estomacal. Desta maneira, recomenda-se a prática do jejum 15 a 18 horas durante o manejo pré-abate, sendo esse procedimento fundamental ao bem-estar dos suínos, à segurança alimentar, à redução dos custos de produção e ao volume de dejetos produzidos.

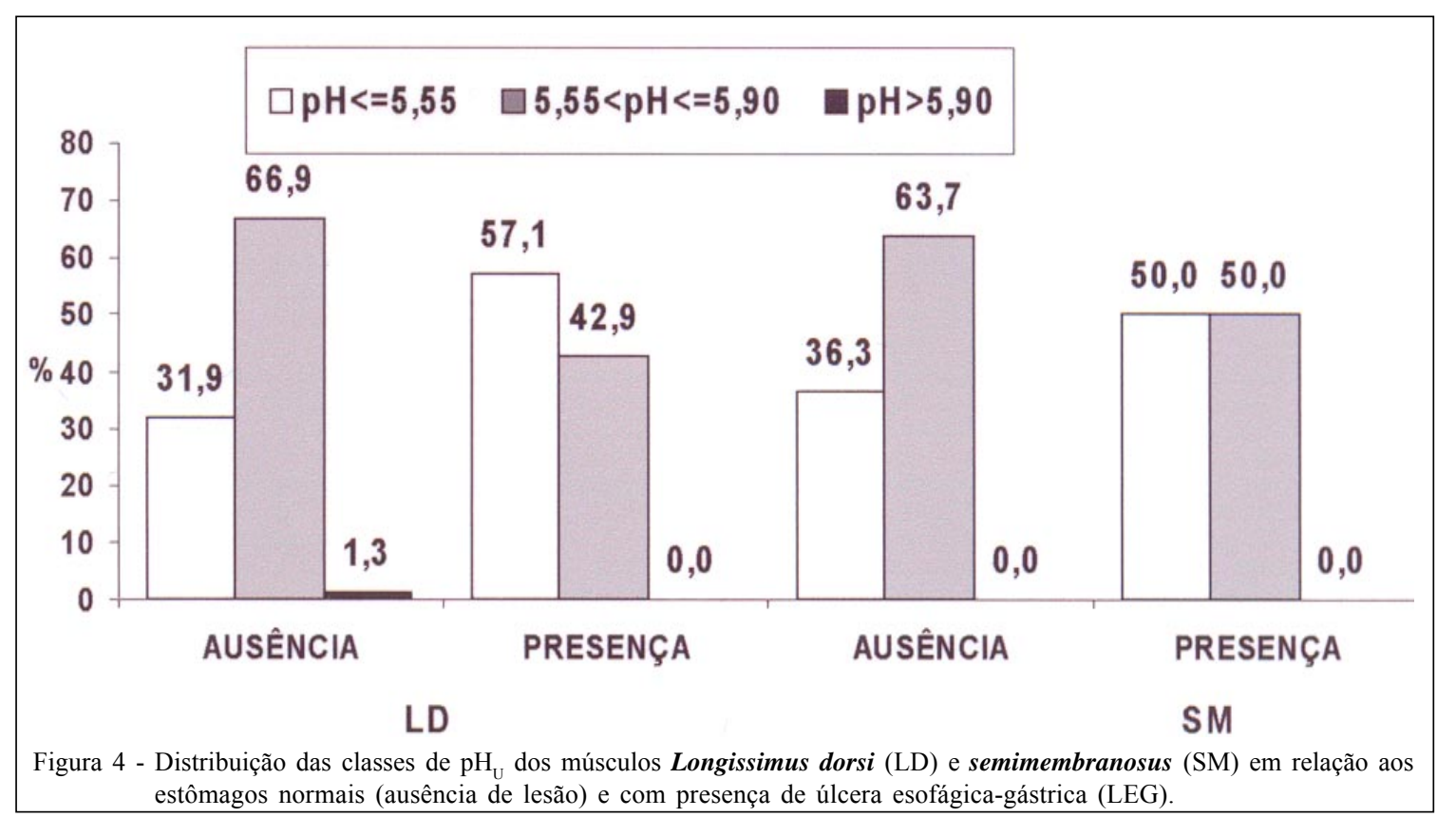

Ciência Rural, v.38, n.1, jan-fev, 2008. 


\section{REFERÊNCIAS}

BEATTIE, V.E. et al. The effect of feed restriction prior to slaughter on performance and meat quality of pigs. Proceedings of Animal Science, v.62, p.11-15, 1999.

BEATTIE, V.E. et al. The effect of food deprivation prior to slaughter on performance, behaviour and meat quality. Meat Science, v.62, p.413-418, 2002.

BIDNER, E.S. The effects of RN geneotype, feed withdrawal prior to slaughter, lysine-deficient diet, and sodium tripolyphosphate pumping on pork quality and sensory characteristics. 1999. 91f. Dissertação (Mestrado em Zootecnia) - University Ollinois.

CHEVILLON, P. Le contrôle des estomacs de porcs à l'abattoir: miroir de la mise à jeun en élevage. Techini-Porc, v.17, p.23$30,1994$.

DALlA COSTA, O.A. et al. Período de descanso dos suínos no frigorífico e seu impacto na perda de peso corporal e em características do estômago. Ciência Rural, v.36, n.5, p.15821888,2006

EIKELENBOON, G. et al. Effects of feed withdrawal before delivery on pork quality and carcass yield. Meat Science, v.29, p.25-30, 1991 .

EISEMANN, J.H. et al. Effect of feed withdrawal prior to slaughter on prevalence of gastric ulcers in pigs. Journal of the American Veterinary Medical Association, v.220, p.503-506, 2002.

GUISE, H. J. et al. Abattoir observations of the weights of stomachs and their contents in pigs slaughtered at known turnes after their last feed. British Veterinary Journal, v. 151 p.659-670, 1995.

JONES, S.D.M. et al. Effects of fasting and water restriction on carcass shrink and pork quality. Canadian Journal of Animal Science, v.65, p.613-619, 1985.

JONES, S.D. M.et al. Body proportions and carcass composition of pigs with known genotypes for stress susceptibility fasted for different periods of time prior slaughter. Canadian Journal of Animal Science, v.68, p.139-149, 1988.

LAWRENCE, B.V. et al. Changes in pars esophageal tissue appearance of the porcine stomach in response to transportation, feed deprivation, and diet composition. Journal Animal Science, v.76, p.788-795, 1998.

LYNCH, P.B. et al. Studies on pre-slaughter handling of pigs and its relationship to meat quality. Acesso em :10 dez 2004. On line. Disponível em: http://www.teagasc.ie/ research/reports/pigs/4130/eopr-4130.pdf.

MAGRAS, C. et al. [Quelles durées de mise à jeun des porcs charcutiers pour um optimun de qualité dês carcasses ? Détermination à partir dún étude terrain]. Journées Recherche Porcine em France, v.32, p.351-356, 2000

MORÉS, N. Influência da granulometria de ingredientes de dietas no desenvolvimento de lesões gástricas em suínos. In: SIMPÓSIO SOBRE GRANULOMETRIA DE INGREDIENTES E RAÇÕES PARA SUÍNOA E AVES, 1998, Concórdia. Anais... Concórdia: EMBRAPA suínos e aves, 1998. p.13-25. (EMBRAPA suínos e aves. Documentos, 52)

MORÉS, N. et al. Avaliação patológica de suínos no abate manual de identificação. Brasília: EMBRAPA Comunicação para Transferência de tecnologia, 2000. 40p.

MORROW, M. et al. The effect of feed withdrawal on pork quality and the prevalence of salmonella and gastric ulcers at slaughter. In: FINAL REPORT TO NATIONAL PORK PRODUCERS COUNCIL. Acesso em: $10 \mathrm{dez} 2004$. On line. Disponível em: <http:/www. porkscience.prg/ documents/research/theeffectoffeed.pdf $>$

MURRAY, C.; JONES, S.D.M. The effect of mixing, fasting and genotype on carcass shrinkage and pork quality. In: INTERNATIONAL CONGRESS OF MEAT SCIENCE AND TECHONOLOGY, 38., 1992, França. Proceedings... França: [s.n.], 1992. p.205-208.

MURRAY, C. Reducing losses from farm gate to packer. Advances in Porks Production, v.11, p.175-180, 2000

MURRAY, C. et al. Effect of preslaughter overnight feed withfrawal on pig carcass and muscle quality. Canadian Journal of Animal Science, v.81, p.89-97, 2001.

SAS INSTITUTE. System for Microsoft Windows: release 8.2. Cary, 2001. 1 CD-Rom

TARRANT, P.V. The last feed before slaughter. Pig International, n.21, p.39-41, 1991

WARRISS, P.D. Loss of carcass weight, liver weight and liver glycogen, and the effects on muscle glycogen and ultimate $\mathrm{pH}$ in pigs Fasted pre-slaughter. Journal of the Science and Food Agriculture, v.33, p.840-846, 1982.

WARRISS, P.D.; BROWN, S.N. The influence of preslaughter fasting on carcass and liver yield im pigs. Livestock Producion Science, v.10, p.273-282, 1983. 